\title{
Mitochondria-associated protein LRPPRC exerts cardioprotective effects against doxorubicin-induced toxicity, potentially via inhibition of ROS accumulation
}

\author{
QUAN TANG, WEI XIONG, XIXIAN KE, JIAN ZHANG, YU XIA and DAXING LIU \\ Department of Cardiac Surgery, Affiliated Hospital of Zunyi Medical University, Zunyi, Guizhou 563006, P.R. China
}

Received January 26, 2020; Accepted July 10, 2020

DOI: $10.3892 / \mathrm{etm} .2020 .9111$

\begin{abstract}
Doxorubicin (DOX) has been widely employed to treat cancer, particularly solid tumors and hematological malignancies, owing to its high efficacy; however, chemotherapy has been indicated to be cardiotoxic and induce adverse effects, including mitochondrial dysfunction and DNA damage, which limits its application. The mitochondria-associated protein leucine-rich pentatricopeptide repeat-containing (LRPPRC) has been reported to serve critical regulatory roles in physiological processes via regulating mitochondrial function. The aim of the present study was to investigate the possible protective effects of LRPPRC against DOX-induced cardiac injury. In a DOX-induced cardiotoxicity model in H9C2 cells, LRPPRC was indicated to be transcriptionally upregulated and stabilize Bcl-2 and Bax. LRPPRC overexpression exhibited protective effects against proliferation and both apoptotic and non-apoptotic cell death following DOX treatment, but not under normal conditions. It was additionally observed that overexpressed LRPPRC reversed the decreases in ATP synthesis, mitochondrial mass and transcriptional activity, which were induced by DOX exposure. Overexpressed LRPPRC also decreased the accumulation of reactive oxygen species (ROS) under DOX treatment and inhibited cell death to a similar extent as $\mathrm{N}$-acetyl-L-cysteine, which is a known ROS scavenger, indicating that LRPPRC potentially exerts protective effects via inhibiting ROS accumulation. Moreover, LRPPRC overexpression protected $\mathrm{H} 9 \mathrm{C} 2$ cells against oxidative stress induced by $\mathrm{H}_{2} \mathrm{O}_{2}$, which also indicated its ROS-scavenging function. The present study demonstrated for the first time, to the best of our knowledge, that DOX-induced LRPPRC may exert cardioprotective effects via inhibiting ROS accumulation, thereby maintaining mitochondrial function.
\end{abstract}

Correspondence to: Professor Daxing Liu, Department of Cardiac Surgery, Affiliated Hospital of Zunyi Medical University, 6 Xuefu West Road, Xinpu, Zunyi, Guizhou 563006, P.R. China

E-mail: daxing1970@163.com

Key words: leucine-rich pentatricopeptide repeat-containing, $\mathrm{H} 9 \mathrm{C} 2$ cells, cardiotoxicity, mitochondrial function, reactive oxygen species, oxidative stress

\section{Introduction}

Doxorubicin (DOX), which is a member of the anthracycline family, has been widely used as a chemotherapeutic agent for solid tumors owing to its effective therapeutic outcomes (1). However, the administration of DOX is limited since anthracycline therapy has been indicated to be closely associated with dose-dependent cardiotoxicity (1). Although the exact molecular mechanism of cardiotoxicity induced by DOX treatment has not fully elucidated, DOX has been reported to promote mitochondrial dysfunction and alter the mitochondrial membrane by eliciting lipid peroxidation, generating free radicals, increasing the myocardial levels of sodium and calcium and inducing autophagy/mitophagy, thereby resulting in apoptotic and non-apoptotic cell death in cardiomyocytes (2-5). DOX-induced toxicity has also been demonstrated to be primarily attributed to the generation of reactive oxygen species (ROS)/reactive nitrogen species (RNS) as a result of drug redox recycling (6). Therefore, decreasing or attenuating DOX-induced ROS accumulation has become a therapeutic strategy to reduce the risk of heart failure (6).

Leucine-rich pentatricopeptide repeat-containing (LRPPRC), which is also known as LRP130, is a well-known mitochondria-associated protein that has been indicated to serve critical roles in mitochondria by maintaining mitochondrial membrane potential and function (7). In the mitochondrial matrix, LRPPRC has been reported to bind stem-loop-interacting RNA-binding protein and subsequently regulate mRNA stability and polyadenylation and the coordination of translation (7-9). Mutations in LRPPRC have been indicated to result in cytochrome $c$ oxidase deficiency, decreased mitochondrial mRNA levels and reduced mitochondrial translation in the liver and brain $(7,10)$. Several reports have revealed that LRPPRC regulates $\mathrm{mRNA}$ transport from the cytoplasm to mitochondria (11) and RNA transport from the nucleus to mitochondria (12); however, LRPPRC has been indicated to be primarily localized in the mitochondria (13). Knockdown of LRPPRC in cells (13) and knockout of Lrpprc in mice (8) has been indicated to reduce mitochondrial membrane potential and homeostasis, and DNA mass and function. Therefore, LRPPRC regulates physiological processes both in vitro and in vivo via regulating mitochondrial function.

Numerous studies have been performed to investigate the effects and the mechanisms by which DOX targets 
mitochondrial energy metabolism and ROS production. Wang et al (14) reported that fibroblast growth factor 21, which is a well-known regulator of glucose and lipid metabolism, exerted cardioprotective effects against DOX-induced toxicity via the NAD-dependent protein deacetylase sirtuin-1/liver kinase B1/AMP-activated protein kinase pathway in $\mathrm{H} 9 \mathrm{C} 2$ cells. Liu et al also reported that pterostilbene, which is a natural analogue of resveratrol and an antioxidant, exerted cardioprotective effects against DOX-induced cardiotoxicity in $\mathrm{H} 9 \mathrm{C} 2$ cells and injury in mouse cardiomyocytes by reducing oxidative stress, including ROS accumulation, which indicated that oxidative stress may be the principal cause of cardiotoxicity following DOX exposure (15). It has also been reported that salsolinol, a plant-based isoquinoline alkaloid, ameliorated cardiomyocyte function, promoting mitochondrial respiratory and energy metabolism (16). However, little is known of the effect of LRPPRC on oxidative stress induced by DOX exposure. The present study aimed to investigate the effects of LRPPRC on $\mathrm{H} 9 \mathrm{C} 2$ cardiomyocytes under DOX-induced oxidative stress, and the regulatory roles of LRPPRC in mitochondrial function.

\section{Materials and methods}

Cell culture and treatment. $\mathrm{H} 9 \mathrm{C} 2$ myoblast cells derived from the rat myocardium were obtained from the Institute of Biochemistry and Cell Biology, Chinese Academy of Sciences. Cells were maintained in DMEM supplemented with 10\% FBS and $10 \mathrm{ml} / 1$ 100X antibiotic-antimycotic solution containing 10,000 units of penicillin and $10 \mathrm{mg} / \mathrm{ml}$ streptomycin at 37 in a $5 \% \mathrm{CO}_{2}$ humidified incubator. All reagents were purchased from Thermo Fisher Scientific, Inc.

For cell treatment, $30 \%$ inhibitory concentration $\left(\mathrm{IC}_{30}\right.$, $4.75 \mu \mathrm{M})$ or $50 \%$ inhibitory concentration $\left(\mathrm{IC}_{50}, 7.16 \mu \mathrm{M}\right)$ of DOX was added into the culture medium for $24,48,72$ and $96 \mathrm{~h}$ at $37^{\circ} \mathrm{C}$ and cells were used for subsequent analysis.

To induce oxidative stress, $200 \mathrm{mM} \mathrm{H}_{2} \mathrm{O}_{2}$ was added into the culture medium for $24 \mathrm{~h}$ at $37^{\circ} \mathrm{C}$ and cells were used for subsequent analysis.

Cell Counting Kit-8 (CCK-8) assay. H9C2 cells were collected and resuspended in serum-free DMEM at a final concentration of $1 \times 10^{6}$ cells $/ \mathrm{ml}$. A total of $6 \times 10^{3}$ cells/well were seeded into a 96-well plate and cultured overnight. The cells were subsequently treated with various concentrations of DOX $(1.0,2.5,5.0,7.5,10.0,12.5,15.0$ and $17.5 \mu \mathrm{M})$ for $24 \mathrm{~h}$ at $37^{\circ} \mathrm{C}$ and CCK-8 solution (Sigma-Aldrich; Merck KGaA) was added to the cells and incubated for between $30 \mathrm{~min}$ and $2 \mathrm{~h}$ at $37^{\circ} \mathrm{C}$ in the dark according to the manufacturer's protocol. The absorbance at $450 \mathrm{~nm}$ was measured using a microplate reader (Synergy 2 Multi-Mode Microplate Reader; BioTek Instruments, Inc.) to determine cell viability.

Reverse transcription-quantitative PCR (RT-qPCR). A total of $1 \times 10^{6} \mathrm{H} 9 \mathrm{C} 2$ cells were employed for RNA extraction using TRIzol $^{\circledR}$ (Invitrogen; Thermo Fisher Scientific, Inc.) following the manufacturer's instructions. Complementary DNA was obtained via reverse transcription using the RevertAid First Strand cDNA Synthesis kit (Fermentas; Thermo Fisher Scientific, Inc.). The reactions were performed according to the following temperature protocol: $65^{\circ} \mathrm{C}$ for $5 \mathrm{~min}$, ice bath for $2 \mathrm{~min}, 37^{\circ} \mathrm{C}$ for $45 \mathrm{~min}$ and $85^{\circ} \mathrm{C}$ for $10 \mathrm{~min}$. qPCR was performed using the SYBR Green Real-Time PCR Master Mixes (Fermentas; Thermo Fisher Scientific, Inc.) according to the following thermocycling conditions: $95^{\circ} \mathrm{C}$ for $2 \mathrm{~min}$; 35 cycles of $95^{\circ} \mathrm{C}$ for $30 \mathrm{sec}$ and $60^{\circ} \mathrm{C}$ for $1 \mathrm{~min}$. The primer sequences used are as follows: LRPPRC forward, 5'-CTG CACTGTGCTCTTCAAGC-3' and reverse, 5'-GACTGCACA CTACCGAAGCA-3'; Bcl-2 forward, 5'-CGACTTTGCAGA GATGTCCA-3' and reverse, 5'-ATGCCGGTTCAGGTACTC AG-3'; Bax forward, 5'-CGAGCTGATCAGAACCATCA-3' and reverse, 5'-CTCAGCCCATCTTCTTCCAG-3'; $\beta$-actin forward, 5'-AGCCATGTACGTAGCCATCC-3' and reverse, 5'-CTCTCAGCTGTGGTGGTGAA-3'; cytochrome $c$ oxidase subunit (COX) 1 forward, 5'-GGAGCAGTATTCGCCATC AT-3' and reverse, 5'-CGACGAGGTATCCCTGCTAA-3'; COX 3 forward, 5'-GAACATACCAAGGCCACCAC-3' and reverse, 5'-TAATTCCTGTTGGGGGTCAG-3'; NADH dehydrogenase subunit 1 forward, 5'-CTCCCTATTCGGAGCCCT AC-3' and reverse, 5'-GGAGCTCGATTTGTTTCTGC-3'; and cytochrome $b$ forward, 5'-GTCGGCGAAGAAAAATGT GT-3' and reverse, 5'-AAGCTGCTCACAGAGGGGTA-3'. $\beta$-actin was employed as an internal standard. Gene expression was calculated using the $2^{-\Delta \Delta \mathrm{Cq}}$ method (17). Each experiment was repeated three times.

MitoTracker Green and MitoTracker Red staining. A total of $1 \times 10^{6} \mathrm{H} 9 \mathrm{C} 2$ cells were loaded with $100 \mathrm{nM}$ green-fluorescing MitoTracker Green (MitoGreen, YEASEN Technology Company, Shanghai) for $30 \mathrm{~min}$ at $37^{\circ} \mathrm{C}$ to measure mitochondrial content, and $500 \mathrm{nM}$ MitoTracker Red (MitoRed, YEASEN Technology Company, Shanghai) for $30 \mathrm{~min}$ at $37^{\circ} \mathrm{C}$. Images were taken using a X71 (U-RFL-T) fluorescence microscope (Olympus Corporation). All data were obtained from experiments with at least three replicates.

Western blot analysis. H9C2 cells were washed 3 times with ice-cold PBS and lysed in RIPA total protein lysis buffer (Guangzhou RiboBio Co., Ltd.) using the SoniConvert ${ }^{\circledR}$ sonicator (DocSense, Chengdu, China) according to the manufacturer's instructions for $3 \mathrm{sec}(\mathrm{s})$ at room temperature. Concentration of protein in the lysate was measured using BCA kit (Sigma-Aldrich; Merck KGaA) and protein was mixed with 5X SDS loading buffer (Beyotime Institute of Biotechnology) and incubated for $10 \mathrm{~min}$ at $100^{\circ} \mathrm{C}$ to denature. Subsequently, $30 \mu \mathrm{g}$ of each proteins was separated on a $6-15 \%$ gradient SDS-PAGE gels and transferred on to a PVDF membrane. Then membrane was blocked in blocking buffer containing 5\% BSA (Beyotime Institute of Biotechnology) in PBS at room temperature for $1 \mathrm{~h}$. Western blotting was performed using the primary antibodies anti- $\beta$-actin $(1: 5,000$; cat. no. ab8226), mouse monoclonal anti-LRPPRC (1:500; cat. no. ab21864), rabbit polyclonal anti-Bcl-2 (1:2,000; cat. no. ab196495) and rabbit monoclonal anti-Bax (1:1,000; cat. no. ab32503; all from Abcam). Membranes were incubated with primary antibodies at room temperature for $1 \mathrm{~h}$. Horseradish peroxidase-conjugated anti-rabbit (cat. no. ab79080) or anti-mouse IgG secondary antibodies (cat. no. ab47827; both 1:20,000; both from Abcam) were then incubated with the membrane at room temperature for a further $1 \mathrm{~h}$. Signals were detected 
using ECL reagent (Thermo Fisher Scientific, Inc.) and Image J software (version 2.0; National Institutes of Health) was used for densitometry. $\beta$-actin was used as the internal reference.

Fluorescence immunostaining. H9C2 $\left(1 \times 10^{5}\right)$ cells attached onto $18 \mathrm{~mm}$ coverslips were fixed with $4 \%$ paraformaldehyde for $10 \mathrm{~min}$ at room temperature and permeabilized with 0.5\% Triton X-100 (Sigma-Aldrich; Merck KGaA) for $10 \mathrm{~min}$ at room temperature. To block unspecific staining, the cells were incubated with $5 \%$ normal goat serum (Thermo Fisher Scientific, Inc.) diluted in $1 \mathrm{X}$ PBS at room temperature for $30 \mathrm{~min}$. Staining was performed using primary LRPPRC antibodies (1:200; cat. no. ab21864; Abcam) at room temperature for $2 \mathrm{~h}$ in the dark, followed by four washes with PBS-Tween 20 $(0.5 \%)$ and incubation with rabbit $\mathrm{Cy} 5{ }^{\circledR}$-conjugated secondary antibody (cat. no. ab6563, 1:2,000 in $0.5 \%$ normal goat serum) for $1 \mathrm{~h}$ at room temperature. Nuclei were subsequently stained with $5 \mu \mathrm{g} / \mathrm{ml}$ DAPI (Sigma-Aldrich; Merck KGaA) at room temperature for $10 \mathrm{~min}$. Images were captured with a X71 (U-RFL-T) fluorescence microscope (Olympus Corporation) at magnification, $\mathrm{x} 100$.

LRPPRC knockdown and overexpression. Knockdown of LRPPRC was achieved via transient transfection of H9C2 cells with small interfering RNA (siRNA) duplexes (cat. no. for si and NC sequence RSH045567; Guangzhou FulenGen Co., Ltd.) targeting LRPPRC mRNA, according to the manufacturer's instructions. A total of $1 \times 10^{6}$ cells in $2 \mathrm{ml}$ serum-free DMEM were seeded per well of 6 -well plates. Transfection complexes were formed via mixing $5 \mu 1$ Lipofectamine ${ }^{\circledR}$ 2000 reagent (Thermo Fisher Scientific, Inc.) with the siRNA oligomer $(50 \mathrm{nM})$ in $0.5 \mathrm{ml}$ OptiMEM medium (Thermo Fisher Scientific, Inc.) at room temperature for $20 \mathrm{~min}$. The complexes were subsequently added to the cells, which were cultured for $48 \mathrm{~h}$ at $37^{\circ} \mathrm{C}$ and collected for subsequent analysis. Control cells were transfected with negative control siRNA.

For LRPPRC overexpression, the coding sequence of rat LRPPRC (accession no. NM_001008519.1) was provided by Guangzhou FulenGen Co. Ltd. and was inserted into the mammalian expressing vector pSG5L-Flag-HA (Addgene, Inc.). Overexpression of LRPPRC was achieved via transient transfection as aforementioned. Notably, for each transfection in 6-well plate, $1.6 \mu \mathrm{g}$ of plasmid was used.

Cell cycle analysis. To analyze the distribution of the cell cycle phases via quantification of the DNA content, H9C2 cells (1x106/well) grown in 6-well plates were washed with ice-cold 1X PBS and fixed overnight at $4{ }^{\circ} \mathrm{C}$ with ice-cold $70 \%$ ethanol. Subsequently, fixed cells were washed 3 times with ice-cold $1 \mathrm{X}$ PBS, collected via centrifugation at $1,000 \times \mathrm{g}$ at $4^{\circ} \mathrm{C}$ for $5 \mathrm{~min}$ and incubated with a final concentration of $100 \mu \mathrm{g} / \mathrm{ml}$ RNase A and $10 \mu \mathrm{g} / \mathrm{ml}$ propidium iodide (PI; Beyotime Institute of Biotechnology) for $15 \mathrm{~min}$ in the dark at room temperature. The cells were analyzed using a three laser Navios flow cytometer (Beckman Coulter, Inc.) and data was analyzed using FlowJo (FlowJo LLC; v9.7.4).

Apoptosis analysis. To detect apoptotic cell death, annexin V-FITC/PI double staining was performed using Annexin V FITC apoptosis detection kit (Becton, Dickinson and
Company) following the manufacturer's instructions. H9C2 cells $\left(1 \times 10^{6}\right)$ were washed with ice-cold $1 \mathrm{X}$ PBS and pelleted by centrifugation at $400 \times \mathrm{g}$ at $4^{\circ} \mathrm{C}$ for $10 \mathrm{~min}$. Following removing of the supernatant, the pellet was resuspended in $1 \mathrm{X}$ binding buffer from the Annexin V FITC apoptosis detection kit and stained with $5 \mu$ l FITC-labeled annexin $\mathrm{V}$ at $4^{\circ} \mathrm{C}$ for $15 \mathrm{~min}$ in the dark. Subsequently, $10 \mu \mathrm{l}$ PI was added to the mixture and incubated at $4^{\circ} \mathrm{C}$ for $5 \mathrm{~min}$ in the dark. The stained cells were analyzed via flow cytometry using a three lasers Navios flow cytometer within $1 \mathrm{~h}$ following staining (Beckman Coulter, Inc.) and data was analyzed using FlowJo.

ATP measurement. To measure total ATP content, $5 \times 10^{5}$ H9C2 cells were suspended and lysed in a buffer containing $0.22 \mathrm{M}$ sucrose, $0.12 \mathrm{M}$ mannitol, $40 \mathrm{mM}$ tricine, $\mathrm{pH} 7.5$ and $1 \mathrm{mM}$ EDTA at $4^{\circ} \mathrm{C}$ for $10 \mathrm{~min}$. The total lysate was analyzed using ATP Bioluminescence Assay kit (Sigma-Aldrich; Merck $\mathrm{KGaA}$ ) according to the manufacturer's instructions and quantitatively measured using the Optocomp I BG-1 luminometer (GEM Biomedical, Inc.).

ROS staining. Subsequently, 2',7'-Dichlorofluorescin diacetate (DCFH-DA; Sigma-Aldrich; Merck KGaA) was used for ROS staining following $4.75 \mu \mathrm{M}$ of DOX exposure at $37^{\circ} \mathrm{C}$ for $24 \mathrm{~h}$. To scavenge induced ROS, $10 \mu \mathrm{M}$ of $\mathrm{N}$-acetyl-L-cysteine (NAC) was added to the culture for $24 \mathrm{~h}$ treatment. Briefly, $1 \times 10^{6} \mathrm{H} 9 \mathrm{C} 2$ cells/well cultured in a 6 -well plate were incubated with $10 \mu \mathrm{M}$ DCFH-DA in DMEM medium at $37^{\circ} \mathrm{C}$ for $20 \mathrm{~min}$. The plates were washed three times with ice-cold $1 \mathrm{X}$ PBS to remove excess DCFH-DA dye. Fluorescence was visualized immediately at $485 \mathrm{~nm}$ (excitation) and $530 \mathrm{~nm}$ (emission) using an inverted fluorescence microscope (Olympus Corporation) at magnification, x100.

Statistical analysis. All data were analyzed using SPSS v13.0 (SPSS, Inc.). Data are presented as the mean \pm standard deviation. The statistical significances of the differences between groups were assessed using unpaired Student's t-test or one-way ANOVA followed by Bonferroni's post hoc analysis. $\mathrm{P}<0.05$ was considered to indicate a statistically significant difference. All experiments were repeated three times independently.

\section{Results}

Low-dose DOX upregulates the expression of LRPPRC. Prior to assessing the effect of DOX exposure on the expression levels of LRPPRC, the cytotoxic effect of DOX on H9C2 cells was measured using the CCK-8 assay. As illustrated in Fig. 1A, the $\mathrm{IC}_{30}(4.75 \pm 0.31 \mu \mathrm{M})$ and $\mathrm{IC}_{50}(7.16 \pm 0.52 \mu \mathrm{M})$ of DOX were employed for subsequent analysis. Following 0, 24, 48, 72 and $96 \mathrm{~h}$ of DOX exposure at $\mathrm{IC}_{30}$ or $\mathrm{IC}_{50}$, the mRNA and protein levels of LRPPRC were detected. The levels of Bcl-2 and Bax, which have been indicated to be regulated by LRPPRC (13), and therefore may be associated with mitochondrial homeostasis, were also examined. Following 24-96 h of DOX exposure at the $\mathrm{IC}_{30}$, LRPPRC mRNA levels were significantly upregulated compared with control cells. By contrast, DOX at the $\mathrm{IC}_{50}$ did not affect LRPPRC, Bcl-2 and Bax mRNA levels, indicating that a low dose of DOX may regulate LRPPRC, and thus modulate mitochondrial function (Fig. 1B). After 48-h 

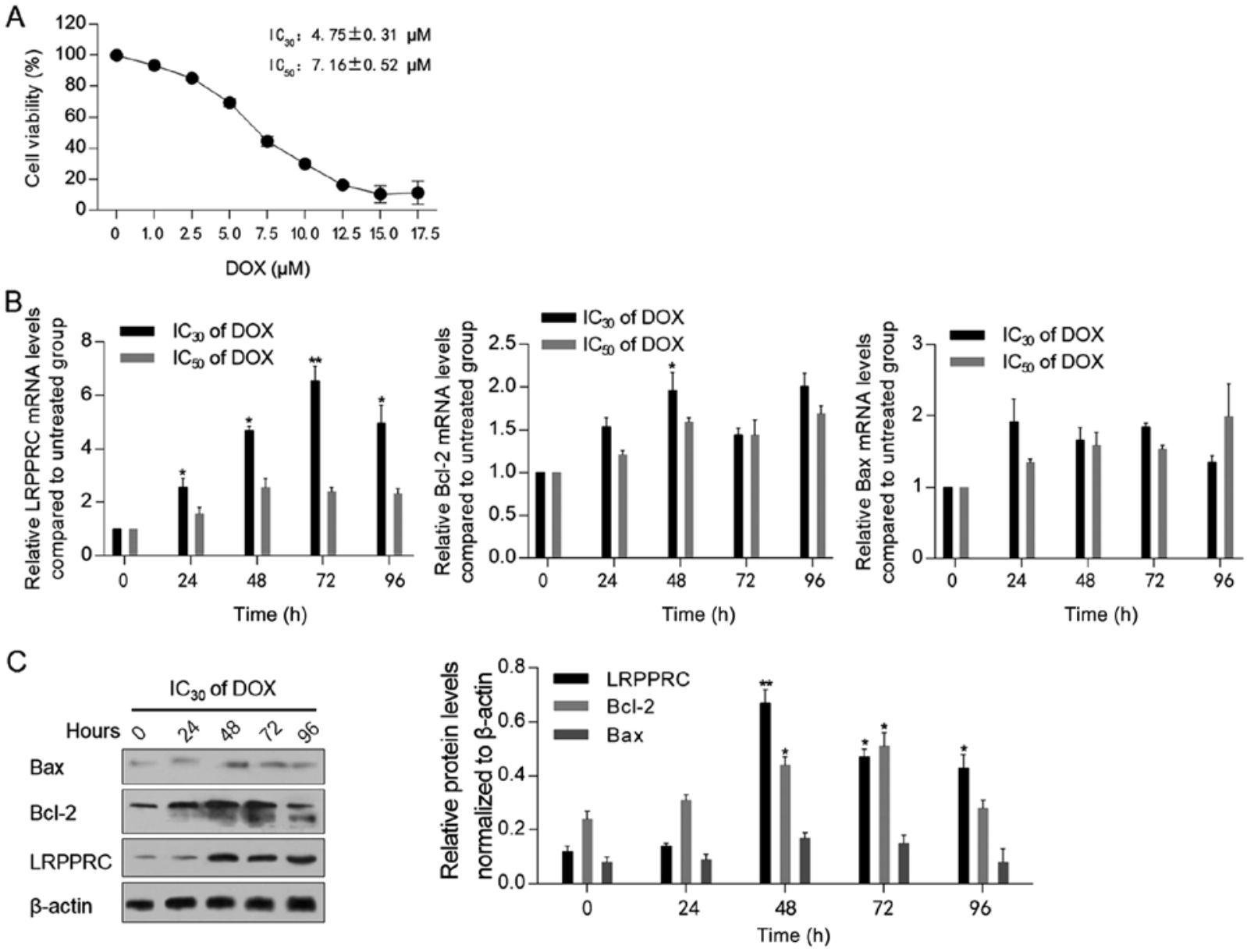

D

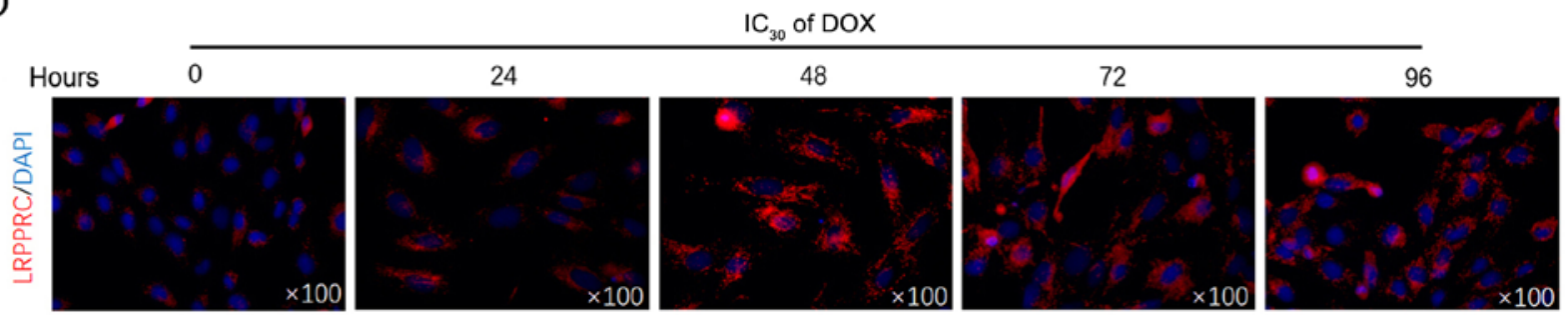

Figure 1. DOX treatment upregulates LRPPRC at the transcriptional level. (A) Cell Counting Kit- 8 assay was performed to detect DOX cytotoxicity in H9C2 cells. (B) Following DOX treatment at $\mathrm{IC}_{30}$ or $\mathrm{IC}_{50}$ for $0,24,48,72$ and $96 \mathrm{~h}$, the mRNA levels of LRPPRC, Bcl-2 and Bax were detected via reverse transcription-quantitative PCR. (C) Protein levels of LRPPRC, Bcl-2 and Bax were detected via western blot (left panel), and quantitatively analyzed via ImageJ software (right panel). (D) Via immunofluorescence staining, the expression and localization of LRPPRC (red) and nucleus (blue) were visualized. ${ }^{*} \mathrm{P}<0.05$ and ${ }^{* *} \mathrm{P}<0.01$ vs. 0 h DOX exposure group. LRPPRC, leucine-rich pentatricopeptide repeat-containing; DOX, doxorubicin.

treatment under $\mathrm{IC}_{30}$ of DOX, Bcl-2 mRNA was upregulated significantly. Subsequently, western blotting was performed to detect the effect of $\mathrm{DOX}$ at $\mathrm{IC}_{30}$ on the protein levels of LRPPRC, Bcl-2 and Bax. In consistence with the alterations at the mRNA level, an increase in the LRPPRC protein expression level was observed following DOX exposure. Notably, the protein levels of Bcl-2 and Bax were increased with no evident increase observed at their mRNA levels, which may be attributed to their possible stabilization by LRPPRC at the protein level (Fig. 1C). A significant increase in Bcl-2 mRNA and protein levels was observed at 48-h exposure, which suggested that $\mathrm{Bcl}-2$ was potentially involved in LRPPRC regulation. The upregulation of LRPPRC following DOX exposure was additionally verified via immunofluorescence staining. As anticipated, the expression of LRPPRC was increased following 24-96 $\mathrm{h}$ of $\mathrm{IC}_{30}$ of DOX exposure compared with control cells (Fig. 1D).

LRPPRC overexpression exerts protective effects against DOX treatment. To assess the effect of LRPPRC under normal conditions and DOX treatment, LRPPRC expression level was firstly modulated via knockdown or overexpression. As demonstrated in Fig. 2A, the LRPPRC expression vector substantially increased the LRPPRC protein level. As knockdown of LRPPRC slightly affected the protein level compared with control cells, which may be attributed to the low endogenous level of LRPPRC, overexpression of LRPPRC was employed for subsequent functional analysis. The CCK-8 assay indicated that overexpression of LRPPRC sensitized H9C2 cells to DOX (Fig. 2B). Under normal conditions, 
A

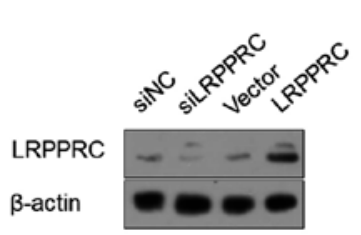

D
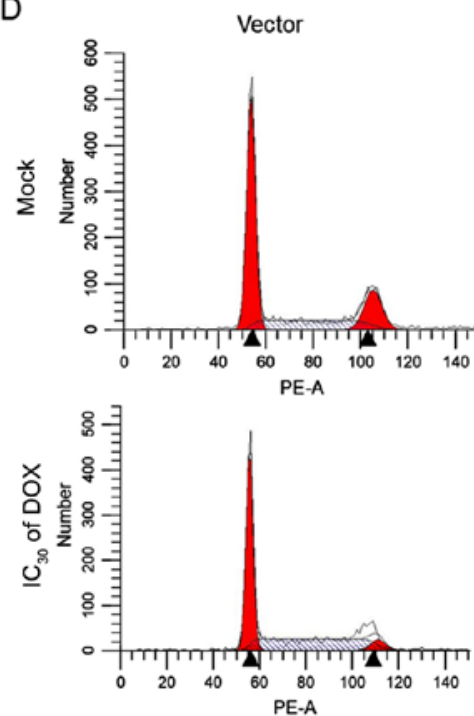

E
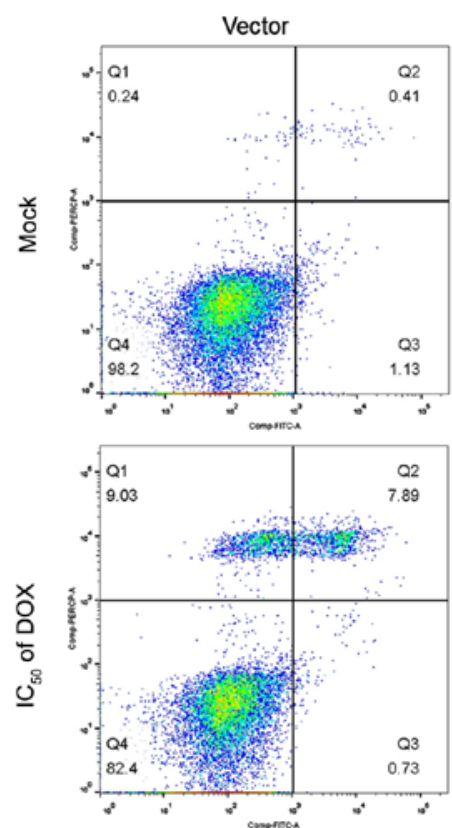
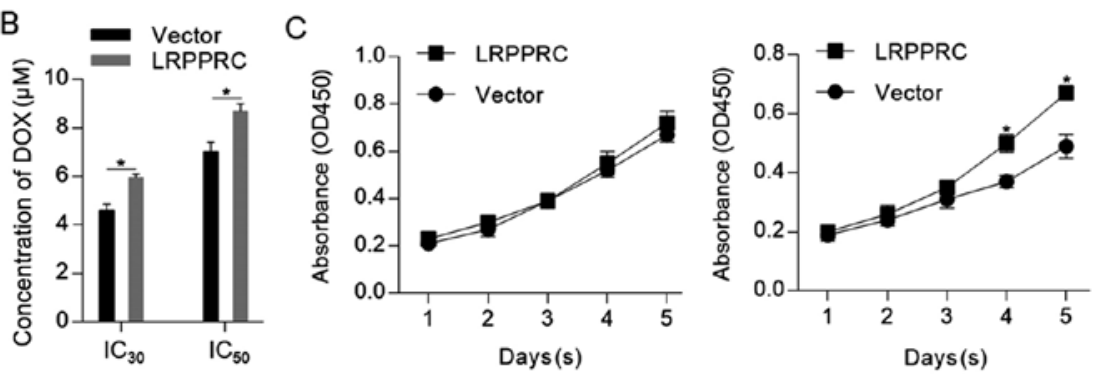

LRPPRC
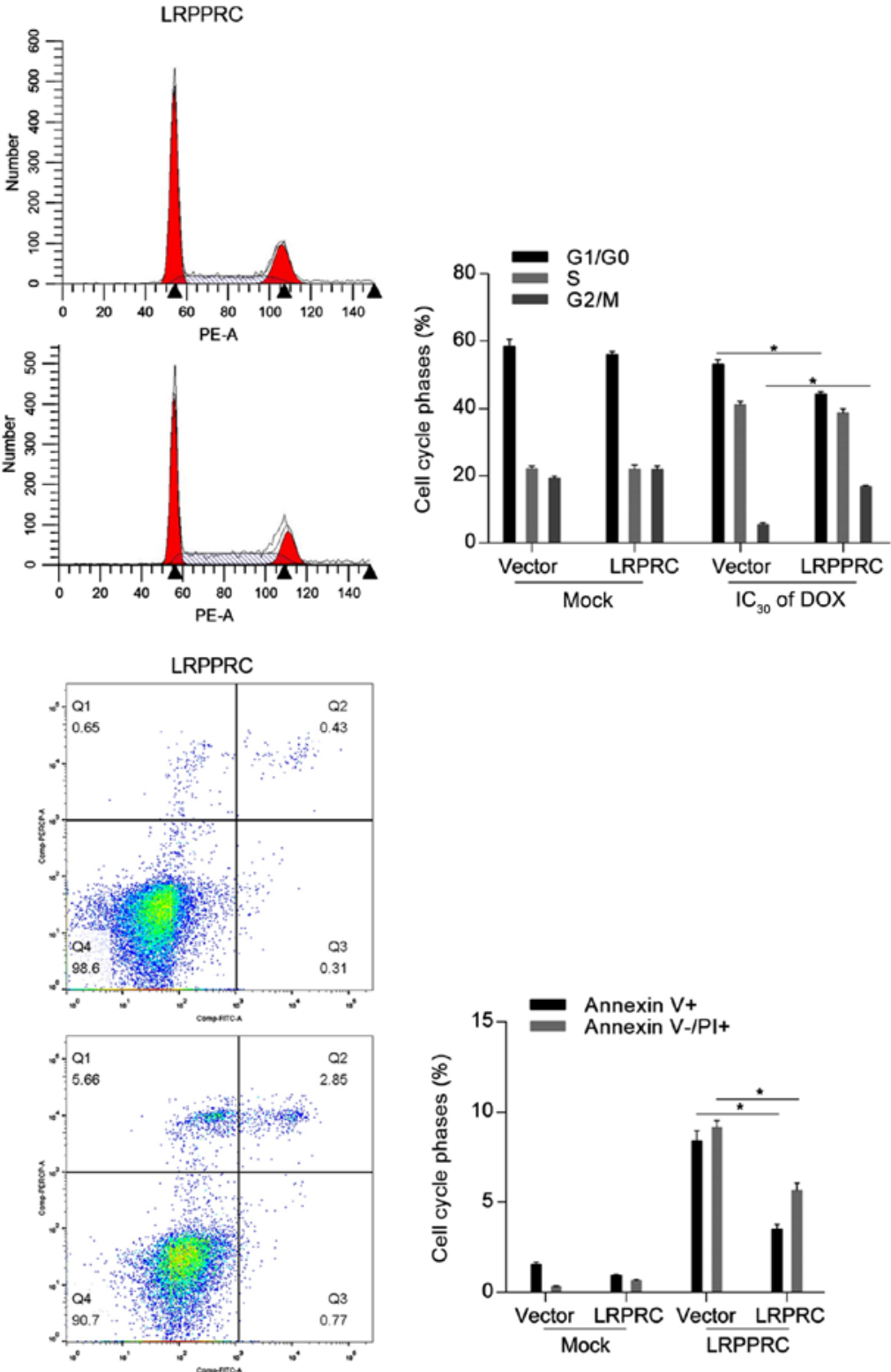

Figure 2. LRPPRC overexpression exerts protective effects against DOX-induced cell injury. (A) A total of $48 \mathrm{~h}$ post-transfection with siLRPPRC and the coding sequence of LRPPRC, the protein levels of LRPPRC were detected via western blotting. (B) Following LRPPRC overexpression, the cytotoxicity of DOX in H9C2 cells was measured. (C) Cell Counting Kit-8 assay was performed to detect the effect of LRPRC overexpression on cell proliferation under DOX treatment. (D) DOX at $\mathrm{IC}_{30}$ was utilized for cell treatment for $24 \mathrm{~h}$, followed by PI staining and flow cytometric analysis to detect the cell cycle phases. (E) DOX at $\mathrm{IC}_{50}$ was employed for cell treatment for $24 \mathrm{~h}$, followed by Annexin V-FITC/PI double staining and flow cytometric analysis to detect apoptotic and non-apoptotic cell death. "P<0.05 vs. vector group. LRPPRC, leucine-rich pentatricopeptide repeat-containing; DOX, doxorubicin; si, small interfering; PI, propidium iodide; OD, optical density.

overexpression of LRPPRC did not affect cell proliferation, while cell proliferation was induced by LRPPRC overexpression following DOX exposure compared with control cells (Fig. 2C). The protective effect of LRPPRC overexpression was additionally verified by detecting the distribution of the cell cycle phases and the apoptotic and non-apoptotic cell death. As illustrated in Fig. 2D and E, although no detectable effects were observed on proliferation and cell death under 
A

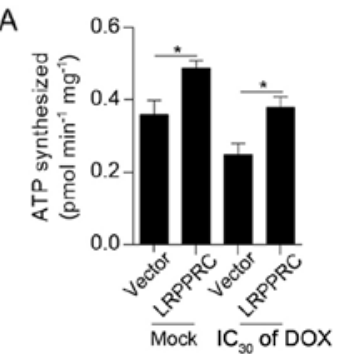

c

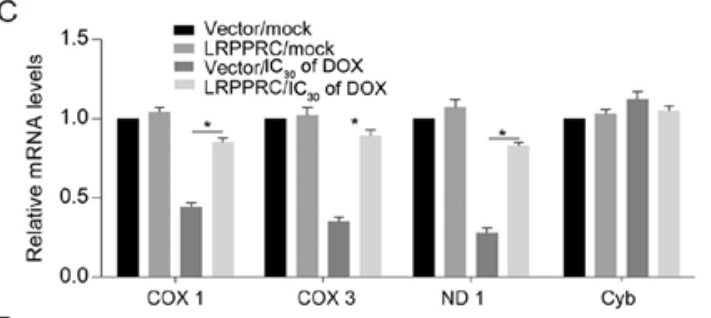

E

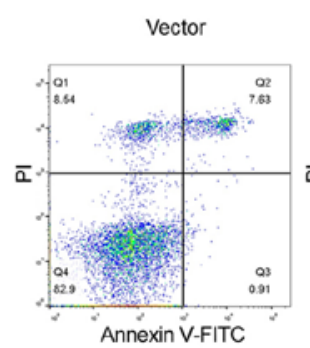

B

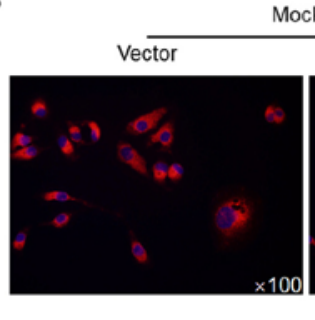

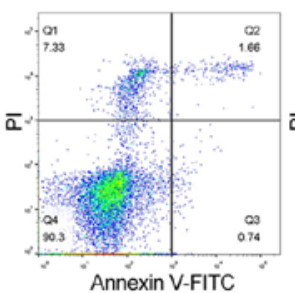

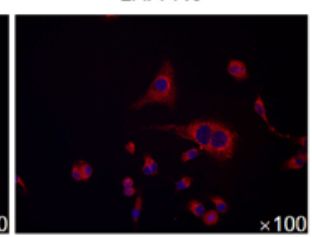

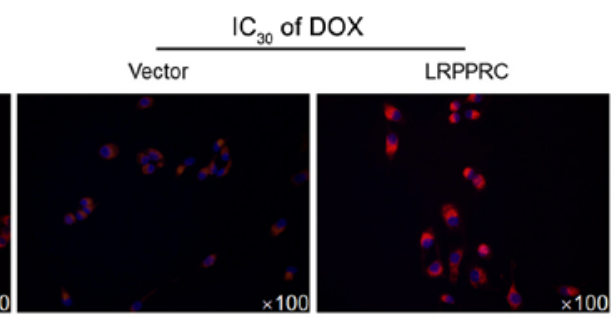

D
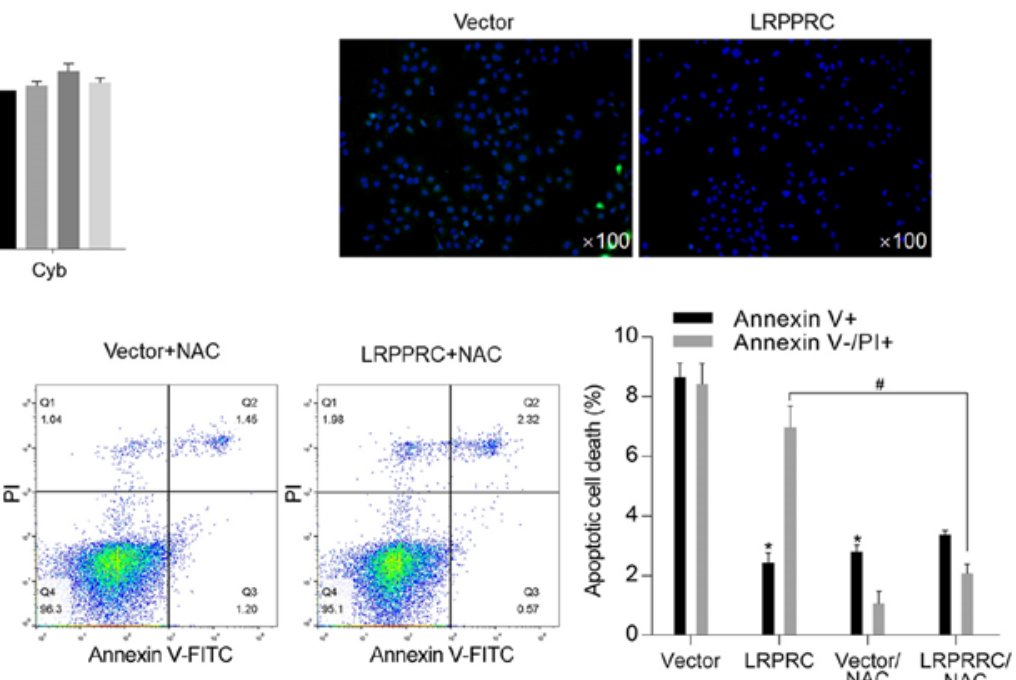

Figure 3. DOX-induced LRPPRC exerts protective effects against DOX exposure potentially via scavenging ROS. (A) Following DOX exposure, the effect of LRPPRC overexpression on ATP synthesis was examined. (B) Mitochondrial mass was measured using MitoTracker Red staining. (C) To evaluate the effect of LRPPRC on mitochondrial transcriptional activity, the expression levels of COX 1, COX 3, ND1 and Cyb were detected via reverse transcription-quantitative PCR. (D) ROS accumulation was detected following DOX exposure at $\mathrm{IC}_{30}$ for $24 \mathrm{~h}$. (E) Annexin V-FITC/PI double staining followed by flow cytometric analysis was performed to detect apoptotic and non-apoptotic cell death. ${ }^{*} \mathrm{P}<0.05$ vs. vector group; ${ }^{~} \mathrm{P}<0.05$ vs. LRPPRC group. LRPPRC, leucine-rich pentatricopeptide repeat-containing; DOX, doxorubicin; PI, propidium iodide; COX, cytochrome $c$ oxidase subunit; ND1, NADH dehydrogenase subunit 1; $\mathrm{Cyb}$, cytochrome $b$; NAC, N-acetyl-L-cysteine; ROS, reactive oxygen species.

normal conditions, overexpression of LRPPRC significantly reversed the DOX-induced $\mathrm{G}_{1} / \mathrm{G}_{0}$ arrest, increased the cell population in the $\mathrm{G}_{2} / \mathrm{M}$ phase and significantly reversed Dox-induced apoptotic and non-apoptotic cell death. Taken together, these results indicated that LRPPRC overexpression exerts protective effects against DOX-induced cytotoxicity of H9C2 cardiomyocytes, while slightly affecting the cells under normal conditions.

LRPPRC overexpression maintains mitochondrial function potentially via scavenging ROS induced by DOX. Considering the critical regulatory roles of LRPPRC on mitochondrial function (7), cellular ATP synthesis was detected following DOX exposure. The results indicated that LRPPRC overexpression significantly increased ATP synthesis both under normal conditions and following DOX exposure (Fig. 3A). Subsequently, mitochondrial function was examined via detecting the mitochondrial mass and transcriptional activity. As illustrated in Fig. 3B and C, the DOX treatment decreased mitochondrial mass and transcriptional activity compared with Mock/vector group, and overexpression of LRPPRC reversed the decrease in mitochondrial mass and transcriptional activity.. Moreover, ROS accumulation was decreased in LRPPRC-overexpressing cells after DOX treatment, which additionally indicated that LRPPRC may exert protective effects via scavenging ROS (Fig. 3D). Following DOX treatment at $\mathrm{IC}_{50}$ for $24 \mathrm{~h}$, DOX-induced cell death was significantly attenuated by overexpression of LRPPRC or ROS scavenger NAC, indicating that LRPPRC potentially regulates ROS accumulation (Fig. 3E).

Accumulated ROS is essential for LRPPRC upregulation. To investigate the mechanism of LRPPRC induction by DOX, it was examined whether accumulated ROS induced by $\mathrm{H}_{2} \mathrm{O}_{2}$ was associated with the upregulation of LRPPRC. As demonstrated in Fig. 4A, treatment with $200 \mathrm{mM} \mathrm{H}_{2} \mathrm{O}_{2}$ for 24 h significantly upregulated the mRNA and protein level of LRPPRC compared with control cells, which was reversed by co-treatment with ROS scavenger NAC. Moreover, NAC attenuated the $\mathrm{H}_{2} \mathrm{O}_{2}$-induced cell cycle arrest and apoptosis (Fig. 4B and C).

\section{Discussion}

The present study investigated the effect of DOX on the expression of LRPPRC, which is a mitochondria-associated regulator, and subsequently evaluated the regulatory effects of DOX-induced LRPPRC on cultured H9C2 cardiomyocytes. DOX has been indicated to exhibit well-known side effects, including the induction of cardiotoxicity, by altering the mitochondrial membrane, which comprises lipid peroxidation, generation of free radicals, 

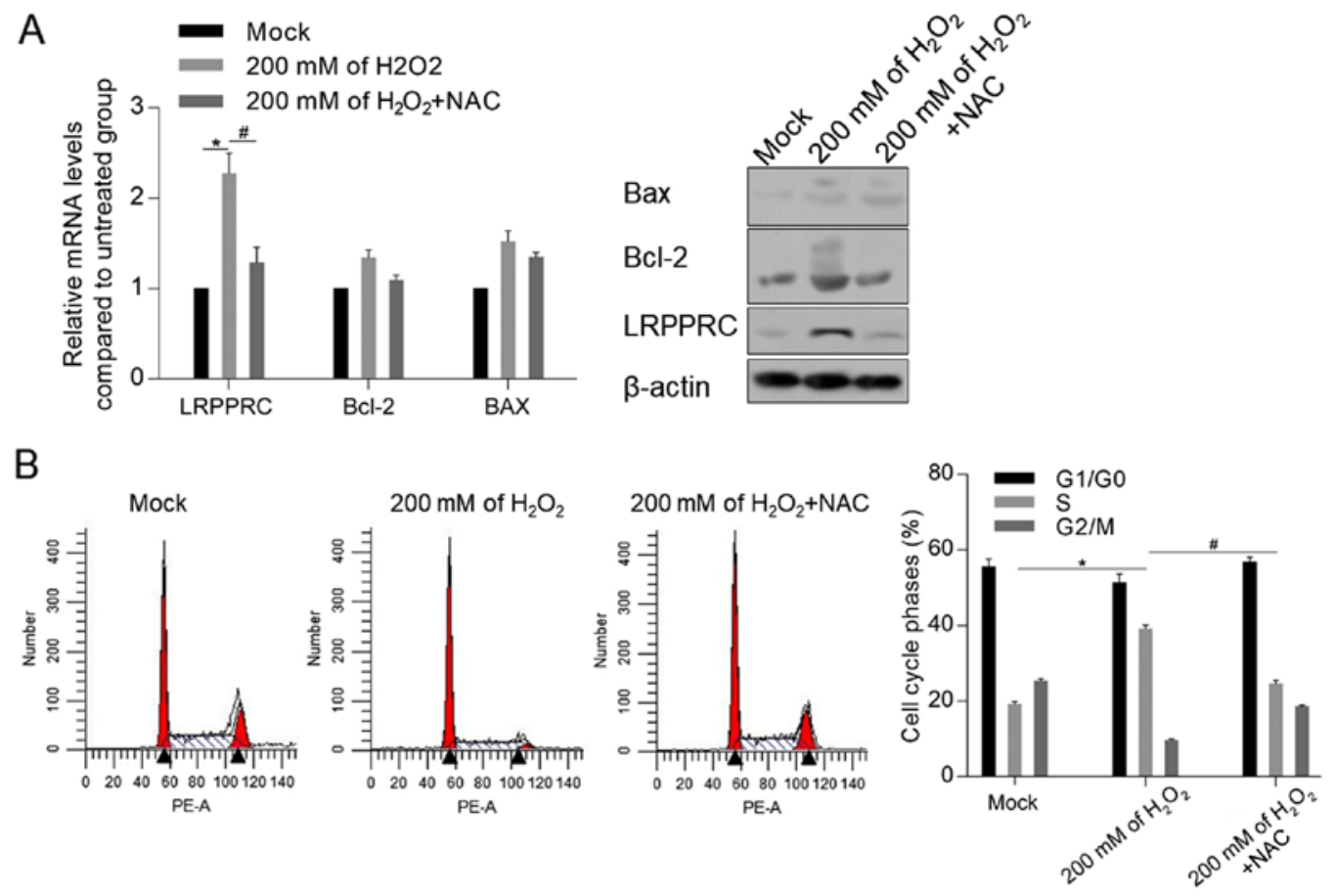

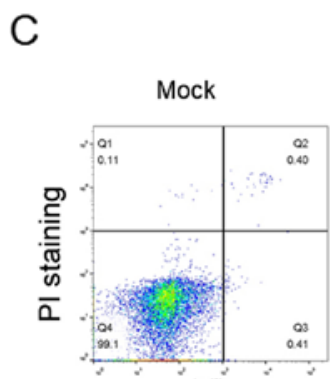

Annexin V-FITC

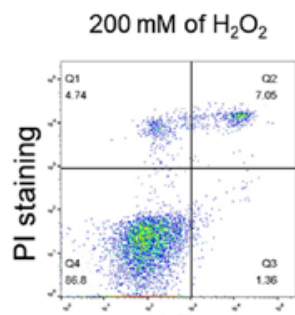

Annexin V-FITC
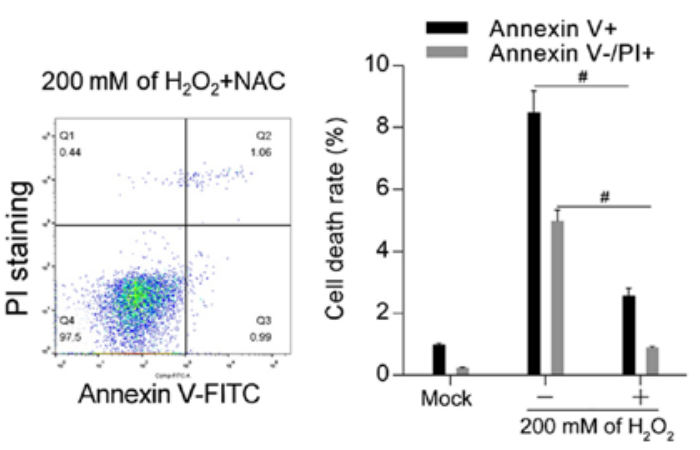

Figure 4. LRPPRC exerts protective effects against reactive oxygen species-induced cell injury in $\mathrm{H}_{9 \mathrm{C} 2}$ cells. (A) Following treatment with $200 \mathrm{mM} \mathrm{H}_{2} \mathrm{O}_{2}$, the mRNA and protein expression levels of LRPPRC, Bcl-2 and Bax were detected. ${ }^{*} \mathrm{P}<0.05$ vs. Mock group; ${ }^{\#} \mathrm{P}<0.05$ vs. $200 \mathrm{mM} \mathrm{H}_{2} \mathrm{O}_{2}$ group. Following $\mathrm{H}_{2} \mathrm{O}_{2}$ exposure with or without NAC treatment, the (B) cell cycle phase distribution and $(\mathrm{C})$ cell death rate were measured. ${ }^{*} \mathrm{P}<0.05$ vs. Mock group; ${ }^{\#} \mathrm{P}<0.05$ vs. $200 \mathrm{mM} \mathrm{H}_{2} \mathrm{O}_{2}$ group. LRPPRC, leucine-rich pentatricopeptide repeat-containing; DOX, doxorubicin; NAC, N-acetyl-L-cysteine; PI, propidium iodide.

the increase in the myocardial levels of sodium and calcium and the induction of autophagy/mitophagy, thereby resulting in apoptotic and non-apoptotic cell death in cardiomyocytes (2-5). The results of the present study demonstrated that DOX induced transcriptional upregulation of LRPPRC, which was followed by stabilization of Bcl-2 and Bax at the protein level. Antioxidants have been indicated to exert cardioprotective effects against DOX exposure via scavenging ROS $(18,19)$. To the best of our knowledge, the current study indicated for the first time that the expression of LRPPRC, which exerted cardioprotective effects against DOX-induced injury, was increased following DOX exposure.

The results of the present study demonstrated that overexpressed LRPPRC exerted protective effects against DOX exposure. LRPPRC reversed the DOX-mediated inhibition of proliferation and cell cycle arrest and improved mitochondrial function. Under normal conditions, overexpressed LRPPRC increased ATP synthesis, mitochondrial DNA mass and transcriptional levels without affecting proliferation, which is consistent with previous findings $(20,21)$. However, overexpressed LRPPRC did not affect cell proliferation and cell cycle distribution under normal conditions, indicating that LRPPRC primarily reversed DOX-induced oxidative stress. To further evaluate the cardioprotective effect of LRPPRC, DOX was administered to cells overexpressing LRPPRC. As anticipated, DOX exposure at a relatively high concentration $\left(\mathrm{IC}_{50}\right.$ of DOX) induced cell death, which was significantly reversed following overexpression of LRPPRC. Consistently, overexpressed LRPPRC did not affect cell death under normal conditions. However, overexpressed LRPPRC significantly inhibited both apoptotic and non-apoptotic cell death induced by DOX treatment, with apoptotic cell death being inhibited to a greater extent. Interestingly, LRPPRC-knockdown did not efficiently reduce LRPPRC levels, which may be attributed to the low levels of endogenous LRPPRC in H9C2 cells.

Mitochondria have been indicated to serve essential roles cardiac cell homeostasis and preventing cells from injury-induced death (22). Dysregulated mitochondrial homeostasis has been reported to induce mitochondrial dysfunction, including a decrease in ATP synthesis, the accumulation of mitochondrial and cytoplasmic ROS and alterations in redox balance in cardiomyocytes (23-25). ROS 
are considered to be the major mediators of DOX-induced cardiotoxicity $(26,27)$. Excess ROS induced by DOX have been demonstrated to result in irreversible mitochondrial damage and exacerbate cardiac diseases (22). In the present study, DOX-induced LRPPRC and overexpressed LRPPRC were revealed to maintain mitochondrial homeostasis and function, as they increased ATP synthesis, mitochondrial mass and transcriptional activity compared with the mock group. Additionally, overexpression of LRPPRC in H9C2 cells reversed DOX-induced ROS accumulation and subsequent apoptotic and non-apoptotic cell death. It was also revealed that accumulated ROS induced by $\mathrm{H}_{2} \mathrm{O}_{2}$ exposure increased LRPPRC protein levels, indicating that ROS may directly regulate LRPPRC. Notably, considering the low endogenous ROS level in the absence of $\mathrm{H}_{2} \mathrm{O}_{2}$, the effect of $\mathrm{H}_{2} \mathrm{O}_{2}$ was specifically detected with or without NAC treatment. Therefore, it was hypothesized that the ROS-LRPPRC axis may form a feedback loop, and it is worth investigating whether accumulated ROS regulates LRPPRC protein level in subsequent studies.

The current study aimed to evaluate the cardioprotective effects of LRPPRC and its function in energy metabolism in H9C2 rat cardiomyocytes. However, certain limitations exist in the present study. Firstly, the cardioprotective effects of LRPPRC were evaluated by introducing exogenous LRPPRC instead of using DOX-induced LRPPRC. Additional studies with DOX pretreatment are required to determine whether DOX-induced LRPPRC exhibits similar effects to those of exogenous LRPPRC. Secondly, although several mitochondrial functions were investigated in the current study (ATP synthesis, mitochondrial transcriptional activity and mass), mitochondria-associated autophagy and mitophagy in $\mathrm{H} 9 \mathrm{C} 2$ cells under DOX treatment still require additional investigation. Taken together, these findings may provide evidence of the cardioprotective effects of LRPPRC against DOX treatment.

\section{Acknowledgements}

The authors would like to thank Mrs. Yun Bai (Third Military Medical University, Chongqing, China) for language editing.

\section{Funding}

The present study was funded by Starting Scientific Foundation of Zunyi Medical University (grant no. SS20180601ZF).

\section{Availability of data and materials}

The datasets used and/or analyzed during the present study are available from the corresponding author on reasonable request.

\section{Authors' contributions}

QT and DL designed the investigation strategy. WX, XK and JZ participated in data collection, performed the statistical analysis and wrote the manuscript. WX and XK performed cell experiments. YX performed part of the molecular experiments, including transfection and RT-qPCR. All authors read and approved the final manuscript.

\section{Ethics approval and consent to participate}

Not applicable.

\section{Patient consent for publication}

Not applicable.

\section{Competing interests}

The authors declare that they have no competing interests.

\section{References}

1. Menna P, Salvatorelli E and Minotti G: Doxorubicin degradation in cardiomyocytes. J Pharmacol Exp Ther 322: 408-419, 2007.

2. Umansky SR, Shapiro JP, Cuenco GM, Foehr MW, Bathurst IC and Tomei LD: Prevention of rat neonatal cardiomyocyte apoptosis induced by simulated in vitro ischemia and reperfusion. Cell Death Differ 4: 608-616, 1997.

3. Olson HM, Young DM, Prieur DJ, LeRoy AF and Reagan RL: Electrolyte and morphologic alterations of myocardium in adriamycin-treated rabbits. Am J Pathol 77: 439-454, 1974.

4. Myers CE, Gianni L, Simone CB, Klecker R and Greene R: Oxidative destruction of erythrocyte ghost membranes catalyzed by the doxorubicin-iron complex. Biochemistry 21: 1707-1712, 1982.

5. Olson RD and Mushlin PS: Doxorubicin cardiotoxicity: Analysis of prevailing hypotheses. FASEB J 4: 3076-3086, 1990.

6. Piasek A, Bartoszek A and Namiesnik J: Phytochemicals that counteract the cardiotoxic side effects of cancer chemotherapy. Postepy Hig Med Dosw (Online) 63: 142-158, 2009 (In Polish).

7. Sasarman F, Brunel-Guitton C, Antonicka H, Wai T and Shoubridge EA; LSFC Consortium: LRPPRC and SLIRP interact in a ribonucleoprotein complex that regulates posttranscriptional gene expression in mitochondria. Mol Biol Cell 21: 1315-1323, 2010

8. Ruzzenente B, Metodiev MD, Wredenberg A, Bratic A, Park CB, Cámara Y, Milenkovic D, Zickermann V, Wibom R, Hultenby $\mathrm{K}$, et al: LRPPRC is necessary for polyadenylation and coordination of translation of mitochondrial mRNAs. EMBO J 31: 443-456, 2012.

9. Chujo T, Ohira T, Sakaguchi Y, Goshima N, Nomura N, Nagao A and Suzuki T: LRPPRC/SLIRP suppresses PNPase-mediated mRNA decay and promotes polyadenylation in human mitochondria. Nucleic Acids Res 40: 8033-8047, 2012.

10. Xu F, Morin C, Mitchell G, Ackerley C and Robinson BH: The role of the LRPPRC (leucine-rich pentatricopeptide repeat cassette) gene in cytochrome oxidase assembly: Mutation causes lowered levels of COX (cytochrome c oxidase) I and COX III mRNA. Biochem J 382: 331-336, 2004.

11. Mili S and Pinol-Roma S: LRP130, a pentatricopeptide motif protein with a noncanonical RNA-binding domain, is bound in vivo to mitochondrial and nuclear RNAs. Mol Cell Biol 23: 4972-4982, 2003.

12. Topisirovic I, Siddiqui N, Orolicki S, Skrabanek LA, Tremblay M, Hoang T and Borden KLB: Stability of eukaryotic translation initiation factor $4 \mathrm{E} \mathrm{mRNA}$ is regulated by $\mathrm{HuR}$, and this activity is dysregulated in cancer. Mol Cell Biol 29: 1152-1162, 2009.

13. Sterky FH, Ruzzenente B, Gustafsson CM, Samuelsson T and Larsson NG: LRPPRC is a mitochondrial matrix protein that is conserved in metazoans. Biochem Biophys Res Commun 398: 759-764, 2010.

14. Wang S, Wang Y,Zhang Z, Liu Q and Gu J: Cardioprotective effects of fibroblast growth factor 21 against doxorubicin-induced toxicity via the SIRT1/LKB1/AMPK pathway. Cell Death Dis 8: e3018, 2017.

15. Liu D, Ma Z, Xu L, Zhang X, Qiao S and Yuan J: PGC1a activation by pterostilbene ameliorates acute doxorubicin cardiotoxicity by reducing oxidative stress via enhancing AMPK and SIRT1 cascades. Aging (Albany NY) 11: 10061-10073, 2019.

16. Wen J, Zhang L, Liu H, Wang J, Li J, Yang Y, Wang Y, Cai H, Li R and Zhao Y: Salsolinol attenuates doxorubicin-induced chronic heart failure in rats and improves mitochondrial function in H9c2 cardiomyocytes. Front Pharmacol 10: 1135, 2019.

17. Livak KJ and Schmittgen TD: Analysis of relative gene expression data using real-time quantitative PCR and the 2(-Delta Delta C(T)) method. Methods 25: 402-408, 2001. 
18. Errami M, Galindo CL, Tassa AT, Dimaio JM, Hill JA and Garner HR: Doxycycline attenuates isoproterenol- and transverse aortic banding-induced cardiac hypertrophy in mice. J Pharmacol Exp Ther 324: 1196-1203, 2008.

19. Hori Y, Kunihiro S, Sato S, Yoshioka K, Hara Y, Kanai K, Hoshi F, Itoh $\mathrm{N}$ and Higuchi S: Doxycycline attenuates isoproterenol-induced myocardial fibrosis and matrix metalloproteinase activity in rats. Biol Pharm Bull 32: 1678-1682, 2009.

20. Mourier A, Ruzzenente B, Brandt T, Kühlbrandt W and Larsson NG: Loss of LRPPRC causes ATP synthase deficiency. Hum Mol Genet 23: 2580-2592, 2014.

21. Cuillerier A, Honarmand S, Cadete V, Ruiz M, Forest A, Deschênes S, Beauchamp C, LSFC Consortium; Charron G, Rioux JD, et al: Loss of hepatic LRPPRC alters mitochondrial bioenergetics, regulation of permeability transition and trans-membrane ROS diffusion. Hum Mol Genet 26: 3186-3201, 2017.

22. Sack MN, Fyhrquist FY, Saijonmaa OJ, Fuster V and Kovacic JC: Basic biology of oxidative stress and the cardiovascular system: Part 1 of a 3-part series. J Am Coll Cardiol 70: 196-211, 2017.

23. Liu D, Ma Z, Di S, Yang Y, Yang J, Xu L, Reiter RJ, Qiao S and Yuan J: AMPK/PGCla activation by melatonin attenuates acute doxorubicin cardiotoxicity via alleviating mitochondrial oxidative damage and apoptosis. Free Radic Biol Med 129: 59-72, 2018.
24. Ma Z, Xin Z, Di W, Yan X, Li X, Reiter RJ and Yang Y: Melatonin and mitochondrial function during ischemia/reperfusion injury. Cell Mol Life Sci 74: 3989-3998, 2017

25. Galley HF, McCormick B, Wilson KL, Lowes DA, Colvin L and Torsney C: Melatonin limits paclitaxel-induced mitochondrial dysfunction in vitro and protects against paclitaxel-induced neuropathic pain in the rat. J Pineal Res 63: e12444, 2017.

26. Takemura G and Fujiwara H: Doxorubicin-induced cardiomyopathy from the cardiotoxic mechanisms to management. Prog Cardiovasc Dis 49: 330-352, 2007.

27. Octavia Y, Tocchetti CG, Gabrielson KL, Janssens S, Crijns HJ and Moens AL: Doxorubicin-induced cardiomyopathy: From molecular mechanisms to therapeutic strategies. J Mol Cell Cardiol 52: 1213-1225, 2012.

This work is licensed under a Creative Commons Attribution-NonCommercial-NoDerivatives 4.0 International (CC BY-NC-ND 4.0) License. 